\title{
Quantitative analysis of connectivity and conductivity in mesoscale multiwalled carbon nanotube networks in polymer composites
}

\section{Citation for published version (APA):}

Gnanasekaran, K., de With, G., \& Friedrich, H. (2016). Quantitative analysis of connectivity and conductivity in mesoscale multiwalled carbon nanotube networks in polymer composites. Journal of Physical Chemistry C, 120(48), 27618-27627. https://doi.org/10.1021/acs.jpcc.6b07458

\section{Document license:}

TAVERNE

DOI:

10.1021/acs.jpcc.6b07458

Document status and date:

Published: 08/11/2016

\section{Document Version:}

Publisher's PDF, also known as Version of Record (includes final page, issue and volume numbers)

\section{Please check the document version of this publication:}

- A submitted manuscript is the version of the article upon submission and before peer-review. There can be important differences between the submitted version and the official published version of record. People interested in the research are advised to contact the author for the final version of the publication, or visit the $\mathrm{DOI}$ to the publisher's website.

- The final author version and the galley proof are versions of the publication after peer review.

- The final published version features the final layout of the paper including the volume, issue and page numbers.

Link to publication

\footnotetext{
General rights

- You may freely distribute the URL identifying the publication in the public portal. follow below link for the End User Agreement:

www.tue.nl/taverne

\section{Take down policy}

If you believe that this document breaches copyright please contact us at:

openaccess@tue.nl

providing details and we will investigate your claim.
}

Copyright and moral rights for the publications made accessible in the public portal are retained by the authors and/or other copyright owners and it is a condition of accessing publications that users recognise and abide by the legal requirements associated with these rights.

- Users may download and print one copy of any publication from the public portal for the purpose of private study or research.

- You may not further distribute the material or use it for any profit-making activity or commercial gain

If the publication is distributed under the terms of Article 25fa of the Dutch Copyright Act, indicated by the "Taverne" license above, please 


\title{
Quantitative Analysis of Connectivity and Conductivity in Mesoscale Multiwalled Carbon Nanotube Networks in Polymer Composites
}

\author{
Karthikeyan Gnanasekaran, Gijsbertus de With,* and Heiner Friedrich \\ Laboratory of Materials and Interface Chemistry, Eindhoven University of Technology, Eindhoven, The Netherlands
}

Supporting Information

ABSTRACT: Mesoscale networks of multiwalled carbon nanotubes (CNTs) dispersed in 50/50 PS/PPO (polystyrene/poly(2,6-dimethyl-1,4-phenylene oxide)) are studied. Various dispersion states are formed by several processing methods such as solvent casting (SC) and extrusion (EXT) and followed by compression molding (CM), which alters the topology of the conductive CNT network. This in turn affects the measured electrical conductivity up to 9 orders of magnitude. Quantitative large-area STEM imaging of the representative topology revealed the existence of subtle differences in topology for various dispersion states. The analyses also reveal that strong localized CNT interactions during the SC process result in highly conductive inhomoge-

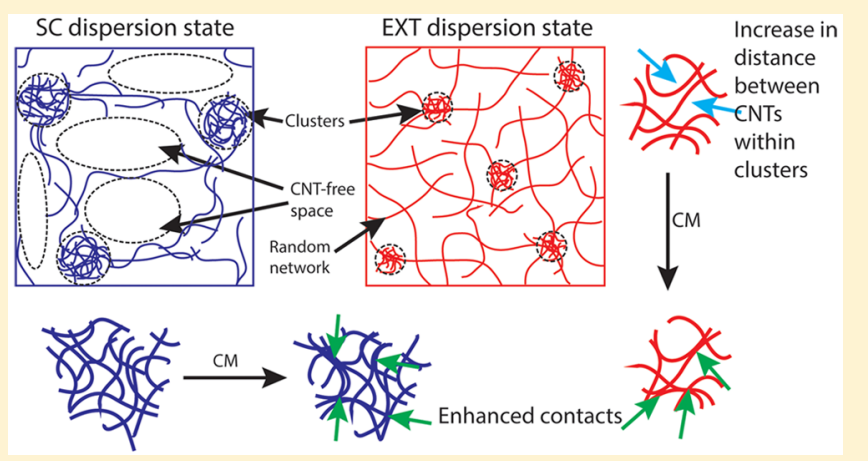
neous networks consisting of CNT clusters connected by individual CNTs embedded in the polymer matrix. This leads to a low electrical percolation threshold, so that as compared to a random network a higher local volume fraction than the overall volume fraction of CNTs is present. A dispersion state created by the extrusion process (EXT) results in less conductive and close to randomly oriented networks. The electrical conductivity of the mesoscale networks is further enhanced by compression molding (annealing), which increases the effective $\mathrm{CNT}-\mathrm{CNT}$ contact area per unit volume and hence decreases the contact resistance between CNTs and results in significant improvement in electrical conductivity. This study illustrates how multiscale information can be used to understand and subsequently tailor the macroscopic functional properties of many scientifically and technologically relevant functional materials.

\section{INTRODUCTION}

Conductive polymer nanocomposites (PNCs) have a high potential to be applied in electrical components. ${ }^{1}$ The electrical conductivity of a conventional polymer, which is commonly insulating, can be increased by several orders of magnitude by incorporating only a very small quantity of conductive fillers like multiwalled carbon nanotubes (CNTs). ${ }^{2,3}$ In such conductive composites the electrical conductivity is strongly influenced, not only by the intrinsic properties of CNTs but also more importantly by the effective charge transport across the CNT network formed within the polymer matrix. ${ }^{4-7}$ Hence, analysis of the formed CNT networks is crucial in evaluating the PNC performance. There are several studies that address the electrical conductivity of PNCs based on statistical changes in network topology resulting from specific processing methods and dispersion states. ${ }^{8-11}$ Nevertheless, for a detailed understanding, representative information on the network topology is required, which is hard to obtain. This is mainly because of the hierarchical structure of the network that extends from the nanometer scale (e.g., size and arrangement of individual CNTs) via the micrometer scale (separation into domains or agglomerates) to the macroscale (inhomogeneities introduced by processing), all affecting the functional proper- ties (mechanical strength, electrical conductivity, and thermal properties). ${ }^{12}$

In this paper, we first prepared different states of such hierarchical structures of CNT networks by various processing methods, such as solvent casting or extrusion, both followed by compression molding. A 50/50 PS/PPO (polystyrene/poly(2,6-dimethyl-1,4-phenylene oxide)) miscible polymer blend is used as matrix material for the preparation of PNCs. Such a polymer blend is used mainly because its composition can be changed further to manipulate their mechanical properties. With the help of quantitative large-scale scanning transmission electron microscopy (STEM), we analyzed the CNT networks of various dispersion states over several tens of micrometers with nanometer resolution, to which we refer to from now on as "mesoscale" analyses. Pegel et al. ${ }^{13}$ applied such stereological and image processing techniques to TEM micrographs to quantify and correlate the dispersion and alignment of CNTs processed by various methods. Here, we show the importance of such quantitative STEM analysis over several length scales, which is required in particular for multiscale morphologies like

Received: July 25, 2016

Revised: November 8, 2016

Published: November 8, 2016 
CNT networks. Such analyses were carried out not only at the electrical percolation region but also in the regime relevant for technological applications, i.e., closer to the plateau in electrical conductivity, and differentiate between the effects of mesoscale ordering (morphology of the network) and nanoscale properties (contact resistance). More importantly, the present study illustrates how the dispersion states can be optimized from the multiscale information to tailor application-specific macroscopic functional properties.

\section{PERCOLATION AND CONDUCTIVITY}

While random percolation is well understood from a theoretical point of view, ${ }^{14}$ one important and intriguing problem in PNC preparation is that the electrical conductivity can be changed drastically, that is, by several orders of magnitude, using different compounding methods. ${ }^{10,12,15}$ Hence, we decided to compare the dispersion states formed by two common preparation methods. In one route, PNCs were prepared by solvent casting of $\mathrm{PS} / \mathrm{PPO}$ and $\mathrm{CNT}$ s using $\mathrm{CHCl}_{3}$ as solvent. The solvent-casted and dried films (SC) were then further processed by compression molding (SC-CM), a method which has been shown to give a percolation threshold much lower than theoretically predicted. ${ }^{16}$ Alternatively, the SC material was melt mixed in an extruder, followed by filament extrusion (EXT). Finally, to complete the series, some of the extruded filaments were also compression molded (EXT-CM). The electrical conductivity curves and percolation of all four PNCs (SC, SC-CM, EXT, EXT-CM) are displayed in Figure 1. More detailed information on preparation, processing methods, and electrical conductivity measurements can be found in the SI, sections I and II.

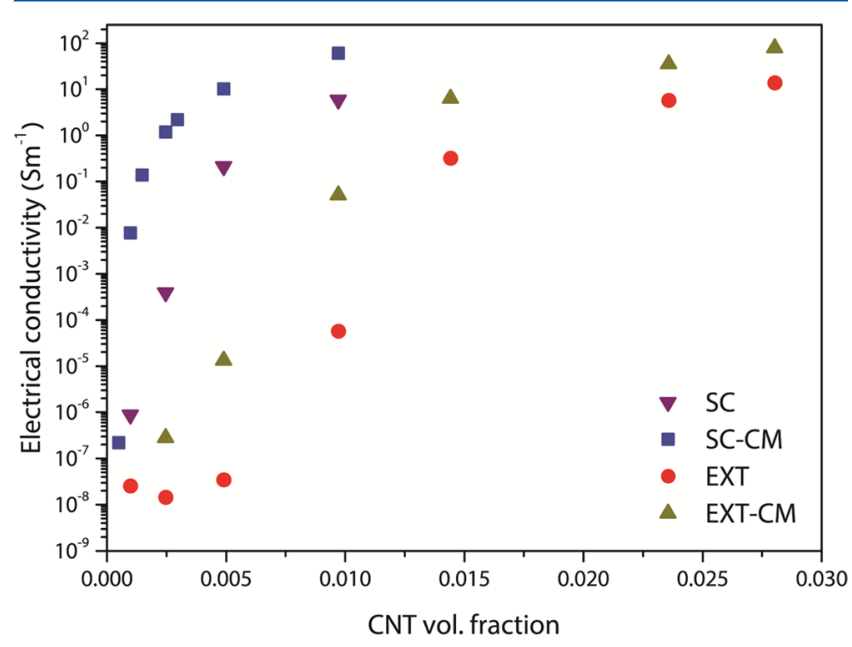

Figure 1. Electrical conductivity plotted as a function of CNT volume fraction for various PNCs.

As expected, all materials show a similar electrical percolation behavior, that is, a steeply increasing electrical conductivity with increasing concentration of CNTs. It is well-known that this steep increase in electrical conductivity-the percolation threshold-occurs when the CNT concentration exceeds a critical value. At the percolation threshold, individual CNTs will make contact with each other (nanoscale) such that a conductive path (mesoscale) is formed, which eventually extends throughout the entire polymer matrix (macroscale). According to percolation theory, the electrical percolation threshold can be lowered using high aspect ratio filler particles, such as CNTs. ${ }^{17}$ Random networks require a good dispersion of the fillers, and in that case the electrical conductivity $\sigma$ is described by

$$
\sigma=\sigma_{c}\left(\frac{\varphi-\varphi_{c}}{1-\varphi_{c}}\right)^{t}
$$

where $\varphi$ is the volume fraction; $\varphi_{\mathrm{c}}$ is the critical volume fraction; and $t$ is a characteristic exponent, depending on the dimensionality of the sample with $t \cong 1.33$ for a $2 \mathrm{D}$ sample and $t \cong 2$ for a $3 \mathrm{D}$ sample. ${ }^{18}$ This expression is valid for $\varphi>\varphi_{\mathrm{c}}$ and for a small value of $\varphi-\varphi_{c}$, while well above the critical volume fraction, the conductivity increases slightly with a linear dependence on volume fraction. ${ }^{19,20}$ From Figure 1 it is already clear that the percolation threshold is vastly different for the networks formed by various processing methods. More importantly, we observe that, starting from the SC process, the percolation threshold is decreased by compression molding and increased by extrusion. The strong influence of compression molding is also reflected by the higher conductivities of the EXT-CM samples in comparison to the EXT samples at the same concentration of CNTs. In summary, there are two mechanisms that affect the conductivity property: (1) mesoscale network formed during the SC and EXT process and (2) nanoscale contacts enhanced by the compression molding process. $^{21}$

Fitting of eq 1 to the conductivities in the percolation region results in $t \cong 2.11 \pm 0.48$ and $\varphi_{\mathrm{c}}=0.00085$ for the SC-CM samples, $t \cong 2.82 \pm 1.01$ and $\varphi_{\mathrm{c}}=0.00947$ for the EXT samples, and $t \cong 1.87 \pm 1.71$ and $\varphi_{\mathrm{c}}=0.00932$ for the EXTCM samples (see SI, section III, for the fitting data). The variation in $t$-value $(t \neq 2)$ has been noticed before, with $t$ ranging from 0.7 to $3.1 .^{18,22,23}$ This variation is observed especially when tunneling is the dominant charge transport mechanism, relating either to a nonrandom microstructure or to complex conduction mechanisms, for example, due to a distribution of resistances that varies with volume fraction. ${ }^{24-27}$ More importantly, the observed variation in percolation threshold by an order of magnitude is rather intriguing and requires a detailed analysis. The percolation threshold obtained by models based on packing parameters (such as volume fraction, aspect ratio) matches closely with the value obtained for extruded materials (EXT) (a detailed discussion on the prediction using different models is provided in the SI, section IV). This close match suggests that the dispersion state formed during the EXT process has a close-to-random network topology.

\section{X-RAY SCATTERING}

It has been shown that the extrusion process can break the $\mathrm{CNTs}^{28}$ and also induce preferential orientation ${ }^{29}$ depending on shear applied in the mixing chamber of the extruder. Analyzing the CNT length distribution after extrusion shows that in our case the distribution remains virtually the same as before extrusion (see SI, section V), probably because of the low shear rate used. To study the orientation of the CNTs in the extruded samples, we performed SAXS experiments. Figures $2 \mathrm{a}, \mathrm{b}$, and $\mathrm{c}$ show the 2D SAXS patterns of PNCs with $\varphi \approx 0.01$ and $\varphi \approx 0.03$ as well as the pure PS/PPO matrix, respectively. Radial averaging of the intensity as a function of $q$ $\left(0.02 \AA^{-1}<q<0.05 \AA^{-1}\right)$ was done, and the resulting intensity was plotted as a function of the azimuthal angle, as shown in Figure $2 \mathrm{~d}$. At percolating volume fraction $(\varphi \approx 0.01)$, the CNT 

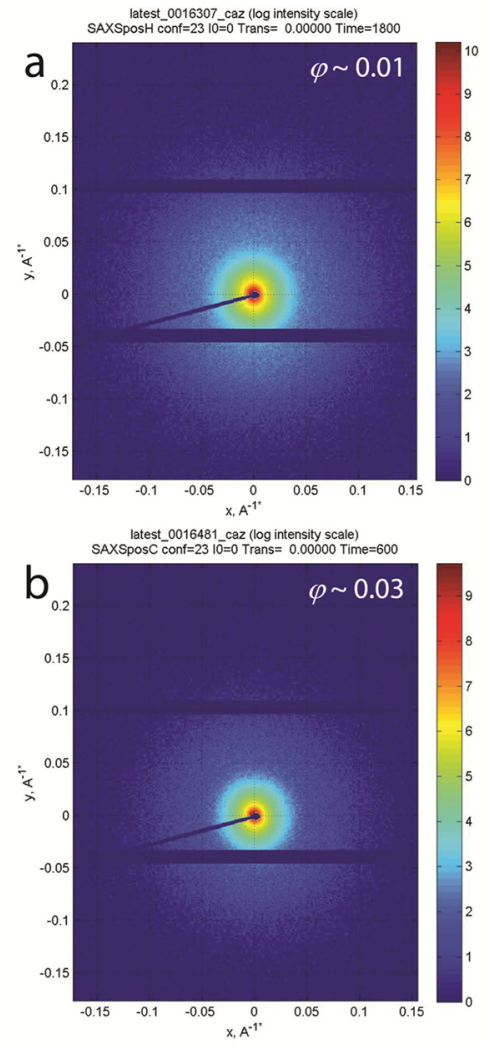

latest_0016485_caz (log intensity scale)
SAXSPosE $\operatorname{conf}=2310=0$ Trans $=0.00000$ Time $=600$
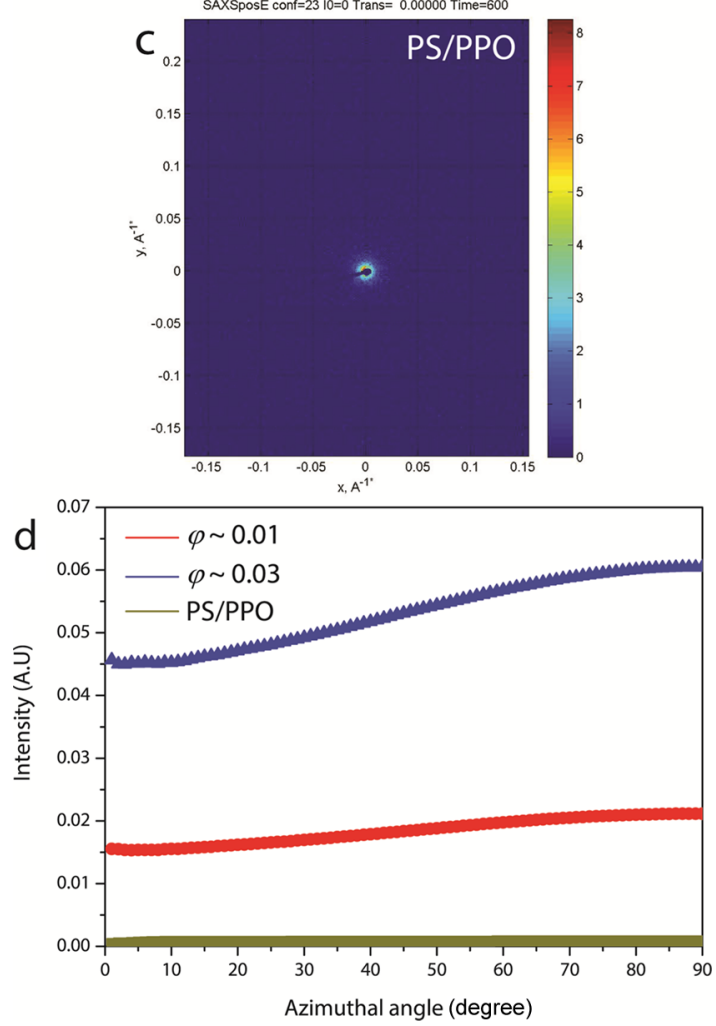

Figure 2. (a,b,c) 2D SAXS pattern of extruded PNCs with $\varphi \approx 0.01$ and $\varphi \approx 0.03$ and the pure PS/PPO matrix. (d) Intensity plotted as a function of azimuthal angle for the corresponding $2 \mathrm{D}$ pattern.

alignment is observed to be rather limited (red curve in Figure 2d) with a full width at half-maximum (fwhm) of $88^{\circ}$, comparable to values reported before. ${ }^{30}$ At higher volume fraction of the CNTs $(\varphi \approx 0.03)$, we observed a small increase in CNT alignment (blue curve in the Figure 2d), also with a fwhm of $88^{\circ}$. Note that in general crystallinity might be induced by the CNTs, thereby affecting the CNT network formation. However, as shown in Figure $2 a-c$, in our case XRD did not reveal any crystallinity.

In addition to the above-mentioned fwhm analysis, the alignment of the CNTs can be quantified by the Herman orientation factor $f$, calculated from the azimuthal intensity distribution, as expressed by ${ }^{31}$

$$
f=\frac{3 \cos ^{2} \phi-1}{2} ; \quad \cos ^{2} \phi=\frac{\int_{0}^{\pi / 2} I(\phi) \cos ^{2} \phi \sin \phi \mathrm{d} \phi}{\int_{0}^{\pi / 2} I(\phi) \sin \phi \mathrm{d} \phi}
$$

The orientation factor $f=1$ when the CNTs are aligned perpendicular to the flow direction, $f=-0.5$ when CNTs are aligned parallel to the flow direction, and $f=0$ for random orientation. We observed $f=-0.032 \pm 0.008,-0.040 \pm 0.003$, and $-0.010 \pm 0.005$ for $\varphi \approx 0.01, \varphi \approx 0.03$, and pure PS/PPO, respectively, and where \pm indicates the standard deviation due to the fit. This clearly illustrates that the distribution of CNTs is basically isotropic in all our samples.

The morphology of the scattered objects can be identified from the scattering pattern as the intensity follows a power law $I(q) \sim q^{-a}$, where $a$ is the fractal dimension varying from 1 to 3 . The fractal dimension $a$ is characteristic for the structure and varies, e.g., 1 for individual rods, 2 for a Gaussian chain and flat disks, and $5 / 3$ for a self-avoiding chain. ${ }^{32,33}$ A slope between 2 and 3 for a chain-like structure, such as CNTs, strongly suggests agglomeration, as disk-like structures for these materials are highly unlikely. We fitted the scattered intensity of the SAXS experiments $\left(0.005 \AA^{-1}<q<0.02 \AA^{-1}\right)$, resulting in $a=2.39$ $(0.03)$ for the PNC with CNT $\varphi \approx 0.01$ and $a=2.40$ (0.03) for $\varphi \approx 0.03$ (and $a=2.92(0.05)$ for the pure PS/PPO matrix). Hence, in our case, the fractal dimension for the PNCs is about 2.4 , indicating that the structure consists mostly of clusters of randomly oriented CNTs. ${ }^{33}$ For higher $q$-values $\left(0.04 \AA^{-1}<q\right.$ $<0.08 \AA^{-1}$ ) a slope $a=1.25$ is obtained, consistent with clusters of curved rods. ${ }^{33}$ In summary, scattering analysis of the PNCs shows that the structure of the PNCs consists largely of clusters of CNTs in which only a slight alignment is present, which contradicts with the percolation models where the extruded samples were shown to have morphology close to a random network of rods without clusters. This discrepancy, an order of magnitude difference in $\varphi_{\mathrm{c}}$ for the SC-CM and EXT samples, and more importantly the fact that statistical percolation theory does not seem to apply to the SC-CM samples call for a detailed investigation of network topology by quantitative large-area STEM analysis. ${ }^{34}$

\section{STEM IMAGING}

One important point in analyzing PNC networks is to realize that they are hierarchical networks, extending from the nanometer to the millimeter scale, which requires analysis of large volumes in order to obtain representative information. We tackle large volume analysis by low convergence angle scanning transmission electron microscopy (STEM) large-area imaging of thick sections, as successfully applied before. ${ }^{34}$ More information on the imaging methods can be found in the SI, section VI. Note that the (S)TEM images represent large-scale overviews but stitched from many high-resolution images, as detailed in ref 34. 


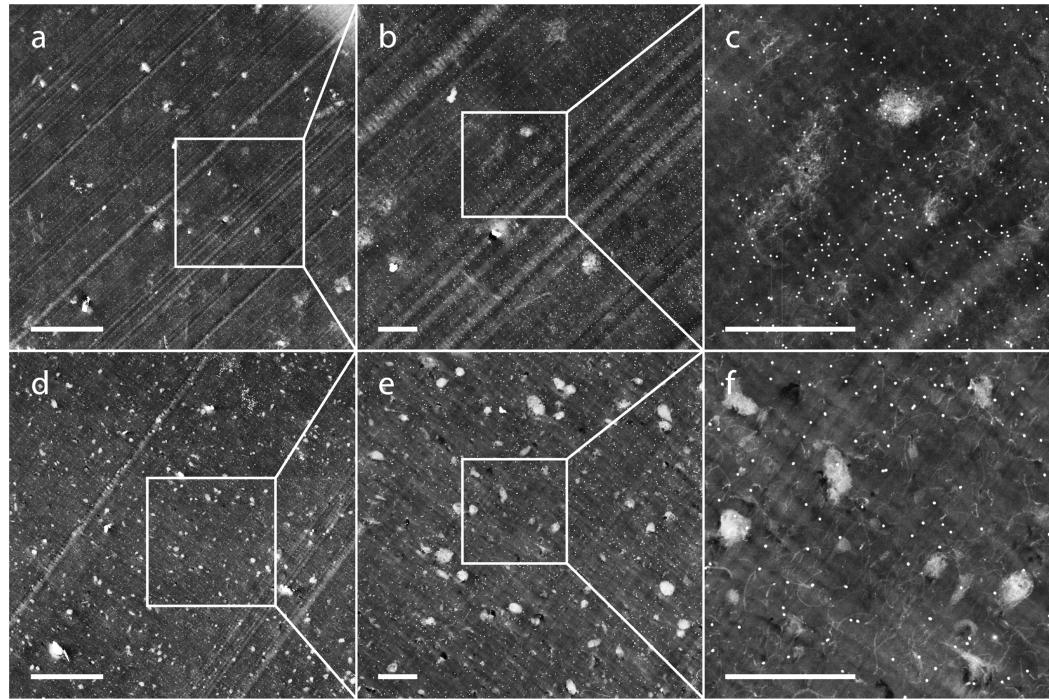

Figure 3. Large-scale STEM images of PNCs. (a,b,c) SC-CM $\varphi \cong 0.01$ and (d,e,f) EXT $\varphi \cong 0.01$. The bright dots are colloidal gold particles which were applied to the sections to aid in stitching of the large-area maps. Scale bars for a and $\mathrm{d}$ are $10 \mu \mathrm{m}$, and $\mathrm{c}$ to $\mathrm{f}$ are $2 \mu \mathrm{m}$.

Large-area STEM maps of SC-CM and EXT PNCs with $\mathrm{CNT} \varphi \approx 0.01$ are shown in Figure 3. Large-area maps of other composites are shown in Figure S3. As expected from the conductivity curves, variations in network topology for both the SC-CM and EXT samples are observed. At all the CNT volume fractions of SC-CM PNCs (Figure $3 a-3 c$ and S3a-S3f), the mesoscale CNT network is observed to be more inhomogeneous with some regions free of CNTs (i.e., filled only with PS/ PPO) and some regions with clusters of CNTs (i.e., a number of CNTs in close contact with each other). The continuity of the network cannot be inferred from the microtome sections, as the length scale of this inhomogeneity exceeds the thickness of the already rather thick STEM sample $(\approx 500 \mathrm{~nm})$. At low volume fraction (close to $\varphi_{\mathrm{c}}$ ), individual CNTs are scarcely visible (Figure S2c) with significant CNT free regions (Figure S3a-S3f). Upon increasing the volume fraction of CNTs to $\varphi$ $\approx 0.01$, the CNTs seem to be randomly distributed and present throughout the entire sample. In addition, clusters are observed to appear (Figure 3a, 3b, and 3c), while similar to the low volume fraction SC-CM samples, localized regions with no trace of CNTs are still present. We suggest that the clusters are formed by a local agglomeration during the SC process and that the resulting structure is preserved during compression molding, as major movement of CNTs during compression molding is unrealistic. ${ }^{35}$ In the case of the EXT samples, the CNTs are distributed homogeneously over the entire field of view at all volume fractions without any sign of CNT-free areas (Figure 3d, 3e, 3f, S3g to S31). This is expected and mainly the result of the shear stress in the extruder during the melt mixing process. Nevertheless, the poor conductivity of the resulting material indicates that a conductive network is not yet formed, in accordance with predictions from percolation theory. In addition to the homogeneously distributed CNTs, small sized clusters are observed, as shown in Figure 3d, 3e, and 3f, which supports the SAXS analysis. These secondary structures could be formed either during drying of the SC films or during the melt mixing process. Although the shear stress induced by the screws disperses the CNTs continuously, keeping most of the CNTs away from each other, some of the CNTs are probably left entangled in the melt.
From the qualitative differences of the various dispersion states of the SC-CM and EXT samples, no apparent differences, in either the volume fraction of CNTs or distribution of CNTs, are obtained. Moreover, the contrast over the polymer phase in the STEM images is rather homogeneous, consistent with the absence of crystallinity. A qualitative examination is therefore not sufficient to link the vastly different conductive properties to the dispersion state. Hence, quantitative image analysis was employed to describe the subtle differences in CNT distribution. To this purpose, first, the representative volume, i.e., the volume over which a measurement provides representative information about the sample, had to be determined, and second, a calibration curve needed to be determined by simulations that translate the CNT area fraction in the image into a CNT volume fraction. Both prerequisites involve the segmentation and quantification of CNTs in the image and are discussed in detail in the SI, sections VII, VIII, IX, and $\mathrm{X}$.

\section{QUANTITATIVE IMAGE ANALYSIS}

Segmented large-area maps illustrating the CNT networks in SC-CM and EXT samples are shown in Figure $4(\varphi \cong 0.01)$ and Figure $S 4(\varphi \cong 0.001-\mathrm{SC}-\mathrm{CM}$ and $\varphi \cong 0.0025-\mathrm{EXT})$. To gain more detailed insight, we quantified the (in)homogeneity of the CNT network by measuring the mean free distance between CNTs, CNT area fraction distribution, and maximum free distance distribution of the CNT-free regions.

The mean free distances $l_{\text {mean }}$ between neighboring CNTs were determined by measuring the mean edge-to-edge uninterrupted distance between all possible pairs of CNTs in the PS/PPO matrix. This is carried out by drawing random lines on the segmented large-area image and measuring the distance between the CNTs, as schematically shown in Figure 5a where 4 different uninterrupted distances $\left(\lambda_{1}\right.$ to $\left.\lambda_{4}\right)$ are indicated. Over 500 such distance values $(\lambda)$ were measured for the SC-CM and EXT PNCs to calculate the mean free distances, the results of which are shown in Figure 5b. For both samples $l_{\text {mean }}$ decreases with volume fraction, but at low CNT volume fraction, $l_{\text {mean }}$ for the SC-CM sample is observed to be higher than for the EXT sample. Upon increasing the CNT 

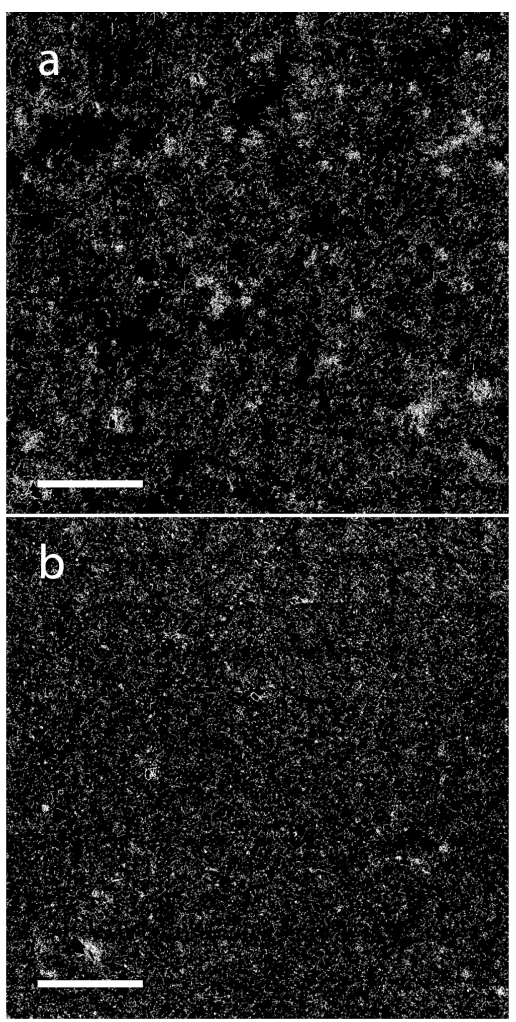

Figure 4. Segmented large-scale STEM images of various PNCs. (a) SC-CM $\varphi \approx 0.01$ and (b) EXT $\varphi \approx 0.01$. Scale bars are $10 \mu \mathrm{m}$.

volume fraction, $l_{\text {mean }}$ for the SC-CM sample becomes smaller than the corresponding value for the EXT sample at $\varphi \cong 0.01$. Though this analysis gives the average description of the topology, it suggests that at $\varphi \cong 0.001$ and $\varphi \cong 0.0025$ the dispersion state is changed from a highly inhomogeneous state with a large part of the material without CNTs formed during the SC process to a less inhomogeneous state by the EXT process. At $\varphi \cong 0.01$, the dispersion state changes from a highly agglomerated state, where most of the CNTs are close to each other, to a relatively less agglomerated state, where the distances between the CNTs are slightly increased by the EXT process. More information can be obtained by measuring the CNT area fraction distribution. To that purpose the largearea STEM maps are divided into segments of $4000^{2}$ pixels, and the CNT area fraction is measured. The distribution of these fractions is shown in Figure 5c (SC-CM) and Figure 5d (EXT). The SC-CM samples have a much broader distribution than the EXT samples at all imaged CNT volume fractions, indicating the presence of much more local variations, i.e., inhomogeneities, in the SC-CM samples. This clear difference in the spatial distribution of CNTs and change in $l_{\text {mean }}$ for the SC-CM samples suggests that the mesoscale network topology changes from the dispersion state with a small amount of CNT-free region to a highly agglomerated dispersion state by increasing the CNT volume fraction. For the EXT samples, the network approaches the dispersion state where the CNTs are homogeneously distribution either from the dispersion state with large amount of CNT-free region or from highly agglomerated dispersion state depending on the CNT volume fraction.

In order to quantify the amount of CNT-free region and agglomeration, the maximum free distance (distribution) of the CNT-free regions is measured; i.e., the distribution of the set of distances that is farthest away from any CNT is measured, and the results are shown in Figure 6. Detailed information on this measurement, labeled as $l_{\text {max }}$ can be found in the SI, section XI. At all volume fractions, the SC-CM samples show a broad and long tail distribution as compared to the EXT samples, reflecting the presence of large and widely distributed CNTfree regions in SC-CM material. This occurs even at higher volume fractions, an effect that is not resolved in the mean free distance measurement (Figure 5). A function describing the distribution of CNT-free regions and the shape of the distribution is formulated as shown below

$$
f\left(l_{\max }\right)=\frac{k}{\lambda}\left(\frac{l_{\max }}{\lambda}\right)^{k-1} \exp \left[-\left(\frac{l_{\max }}{\lambda}\right)^{k}\right]
$$

where $k$ represents the shape of the distribution and $\lambda$ is a scale parameter that represents the width of the distribution. The fit of eq 3 to our data points is shown in Figure S12, and the values of $\lambda$ and $k$ obtained are shown in Table 1 . At all volume fractions, the $k$-values of the EXT samples (average $k \cong 1.68$ ) are different from those of the SC-CM samples (average $k \cong$ 1.34). This elucidates that the CNT-free region changes more gradually (long tails) for the SC-CM samples, irrespective of the volume fraction. More importantly, with an increase in CNT concentration, the $k$-value increases for the SC-CM samples and decreases for the EXT samples. This illustrates that the effective change in topology by moving from solvent-casted material to extruded material is significantly higher at lower volume fractions and that this change becomes smaller at the highest CNT concentration. This significant difference in network at low CNT volume fraction results in several orders of magnitude difference in conductivity, as shown in Figure 1. An important fact that cannot be assessed via the $k$-value is whether the small effective change in topology at high CNT concentration is due to the formation of clusters or just due to the large amount of CNTs. The decrease in $\lambda$ by increasing the concentration of CNTs shows that the network changes from a wider distribution at lower CNT volume fraction to a narrower distribution at higher CNT volume fraction. While comparing the SC-CM samples and EXT samples, the differences in $\lambda$ of the CNT-free region are $44 \%, 28 \%$, and $9 \%$ for $\varphi \cong 0.001, \varphi \cong$ 0.0025 , and $\varphi \cong 0.01$, respectively. From the resulting increase in $l_{\text {mean }}$ for the EXT samples at $\varphi \cong 0.01$, we inferred that the topology changes from a more agglomerated state to a less agglomerated state upon extrusion; however, the difference in $\lambda$-value for the CNT-free space decreases to $9 \%$. This suggest the CNTs are agglomerated and distributed as clusters. Upon extruding, the distance between CNTs within the clusters increases, which is revealed by the increase in $l_{\text {mean }}$ and the distance between the clusters decreases, which is revealed by the decrease in the value of $\lambda$.

To obtain quantitative information on the CNT clusters and CNT-free regions, we classified the $l_{\max }$ data with $l_{\max }>\langle L\rangle / 2$, where $\langle L\rangle$ represents the average CNT length, as CNT-free regions, and the data with $l_{\max }<50 \mathrm{~nm}$ as CNT clusters. Table 2 shows the amount of CNT-free regions and clusters, as quantified by our criteria, and it indicates the presence of more free space for the SC-CM than for the EXT samples. For instance, it shows the presence of almost $47 \%$ of CNT-free space in percolating SC-CM samples $(\varphi \approx 0.001)$; i.e., $47 \%$ of the PS/PPO matrix does not have any CNTs in a region with diameter equal to the average length of CNTs. The amount of CNT-free regions is negligible in the EXT samples at $\varphi_{\mathrm{c}} \approx 0.01$, 

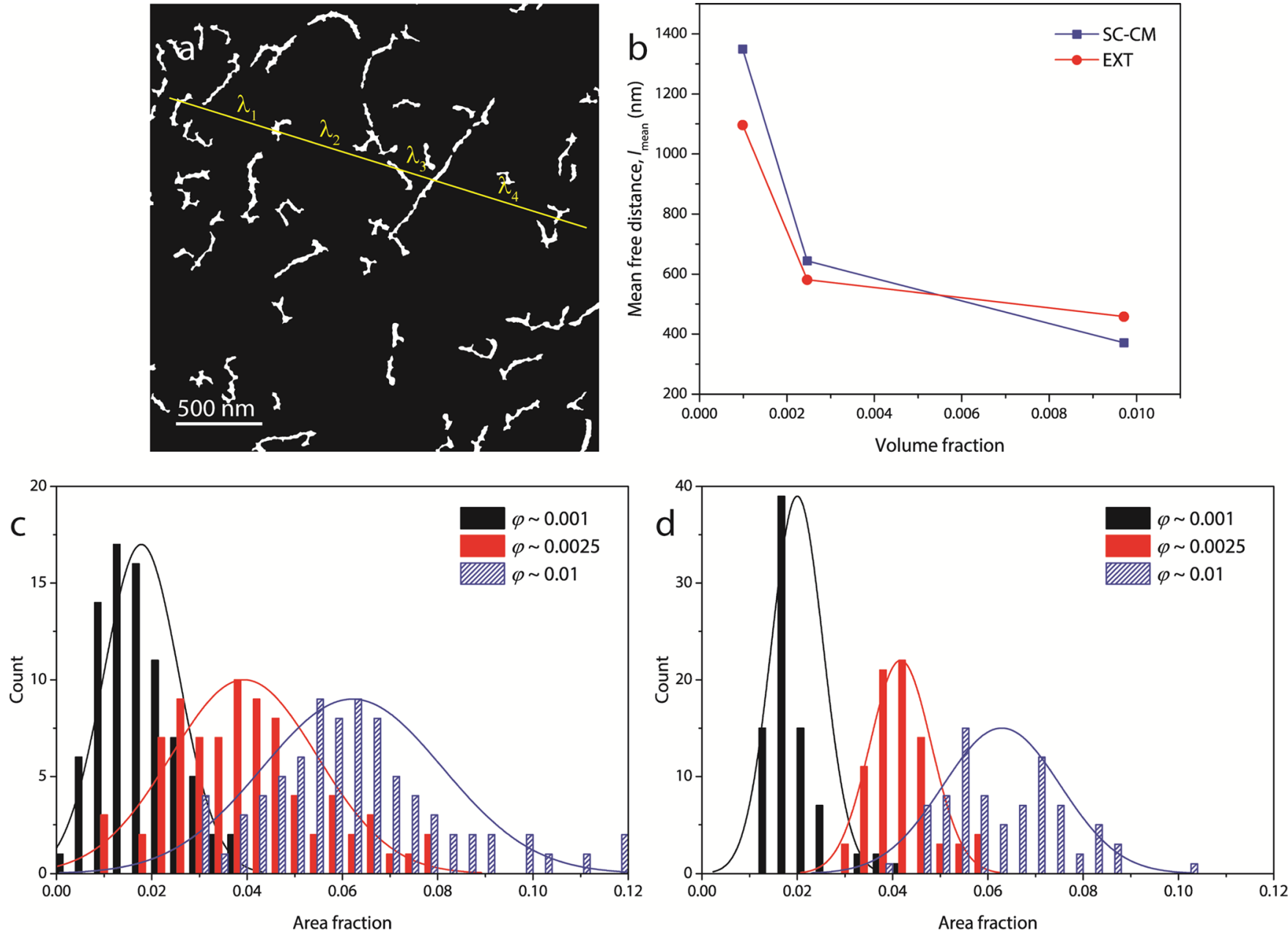

Figure 5. (a) Schematic illustration of the mean free distance measurement on large-scale STEM images. Scale bar is $500 \mathrm{~nm}$. (b) Mean free distance $l_{\text {mean }}(\mathrm{nm})$ as a function of volume fraction of CNTs. (c) Distribution of CNT local area fractions in SC-CM samples and (d) distribution of CNT local area fractions in EXT samples. Image analyses were carried out on a field of view of $(40000)^{2}$ pixels of data acquired from $536 \mathrm{~nm}$ thick sections.

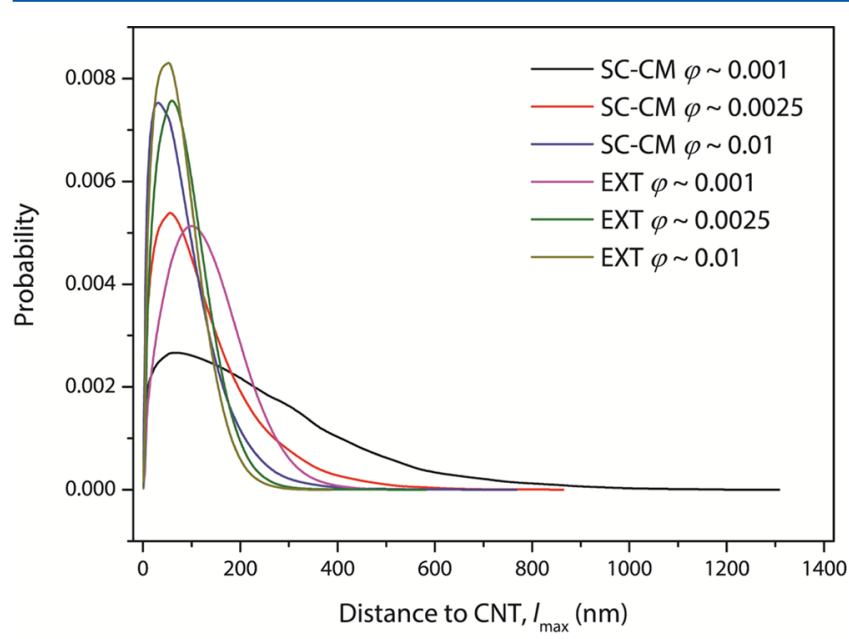

Figure 6. Normalized maximum free distance distribution $\left(l_{\max }\right)$ of the CNT-free regions for the SC-CM and EXT samples.

while the same amount of CNT clusters is observed for both the SC-CM and EXT samples for all volume fractions. This shows that the amount of clusters increases with increasing the CNT concentration, and they remain intact even after the extrusion process. However, the size of the clusters in EXT samples is smaller than in SC-CM samples, indicating that the primary clusters formed during the solvent casting process break apart by the shear involved in extrusion process and form secondary clusters in EXT samples (Figure 2f). We tentatively conclude that the combination of these clusters and CNT-free regions formed by the local agglomeration process results in a highly conductive mesoscale network in the SC samples (SC and SC-CM) compared to the EXT samples, as detailed below.

\section{DISCUSSIONS ON THE DIFFERENCES IN CONDUCTIVE NETWORKS}

Based on the above quantitative analysis, we propose a model describing the differences in the conductive properties of SC and EXT networks, as illustrated in Figure 7 with a simplified schematic containing both the meso- and nanoscale aspects. For each CNT volume fraction, both the SC-CM and EXT networks contain almost the same amount but differently sized CNT clusters, connected by dispersed CNTs. In addition, the SC samples contain CNT-free regions (Figure 7, left). In SC samples an inhomogeneous network is formed due to the local agglomeration process that effectively increases the free space between dispersed CNTs. This in turn leads to a significant decrease in percolation threshold ${ }^{36-39}$ (Figure 7, middle), as the inhomogeneous network appears to have a higher effective 
Table 1. Fitting Parameters of Equation 3

\begin{tabular}{|c|c|c|c|c|c|c|}
\hline & \multicolumn{2}{|c|}{$\varphi \approx 0.001$} & \multicolumn{2}{|c|}{$\varphi \approx 0.0025$} & \multicolumn{2}{|c|}{$\varphi \approx 0.01$} \\
\hline & SC-CM & EXT & SC-CM & EXT & SC-CM & EXT \\
\hline$k$ & $1.281 \pm 0.003$ & $1.778 \pm 0.005$ & $1.360 \pm 0.006$ & $1.670 \pm 0.007$ & $1.374 \pm 0.007$ & $1.588 \pm 0.01$ \\
\hline$\lambda$ & $274.28 \pm 7.16$ & $153.13 \pm 4.42$ & $138.63 \pm 6.28$ & $100.09 \pm 3.88$ & $96.54 \pm 4.35$ & $87.98 \pm 4.73$ \\
\hline
\end{tabular}

Table 2. CNT-Free Regions, Amount of Clusters (in Area $\%)$, and the Effective Increase in CNT Volume Fraction $\varphi_{\text {inc }}$ in SC-CM and EXT Samples at Various Volume Fractions $\varphi$ Using 536 nm Thick Sections

\begin{tabular}{|c|c|c|c|c|c|c|}
\hline & \multicolumn{2}{|c|}{$\varphi \approx 0.001$} & \multicolumn{2}{|c|}{$\varphi \approx 0.0025$} & \multicolumn{2}{|c|}{$\varphi \approx 0.01$} \\
\hline & SC-CM & EXT & SC-CM & EXT & SC-CM & EXT \\
\hline CNT-free regions & 46.9 & 13.2 & 16.5 & 1.8 & 5.1 & 0.80 \\
\hline clusters & 6.3 & 6.1 & 12.0 & 13.4 & 19.9 & 18.1 \\
\hline$\varphi_{\text {inc }}(\%)$ & 88.2 & 15.2 & 19.8 & 1.8 & 5.3 & 0.8 \\
\hline
\end{tabular}

CNT volume fraction as compared with a random network (Figure 7, right) that is observed in EXT samples.

The significant amount of CNT-free space representing only the PS/PPO matrix material in the SC-CM samples characterizes the network formed during the solvent evaporation process, as it is unrealistic to imagine massive movement of CNTs during the compression molding. ${ }^{35}$ As stated above, this suggests that the effective volume fraction of CNTs for the SC samples is higher than the average volume fraction and indicates significant $\mathrm{CNT}-\mathrm{CNT}$ interactions and attachment during network formation. By subtracting the CNT-free fraction, we quantified the effective increase in CNT volume fraction $\varphi_{\text {inc }}$ as shown in Table 2 . In the percolating SC-CM sample $(\varphi \approx 0.001), \varphi$ is increased by $88 \%$. Such an increase is not obtained for the EXT samples with the same volume fraction because they contain, apart from a certain amount of clusters, a homogeneous, random distribution of CNTs (Figure 7, right), resulting in an insulating sample at this CNT concentration.

Still, it is difficult to understand that by an increase of $88 \%$ in CNT volume fraction we achieved percolation in the SC-CM samples resulting in several orders of magnitude increase in conductivity. However, in general, to be representative for the volume, such a free volume analysis in $2 \mathrm{D}$ should be carried out on cross sections. ${ }^{40,41}$ As our analysis was based on projected images, to reduce the projection error, we also carried out largearea maximum free distance distribution analysis on a series of sections with varying thickness to quantify the CNT-free volume. The effective CNT-free volume increase $\Delta \varphi$ (for SC$\mathrm{CM} \varphi \approx 0.001$ ) was estimated for section thicknesses $t$ of 121 $\mathrm{nm}, 219 \mathrm{~nm}, 536 \mathrm{~nm}$, and $926 \mathrm{~nm}$, leading to $\Delta \varphi=81.2 \%$, $65.5 \%, 46.9 \%$, and $30.2 \%$, respectively, as shown in Figure 8 . While the thinnest section leads to an effective CNT-free volume increase of $81.2 \%$, exponential extrapolation to zero thickness leads to $89.2 \%$. This corresponds to an effective increase in CNT volume fraction $\varphi_{\text {inc }}$ in other regions of the composite by a factor of 5.3 and 9.1, respectively, closely matching the observed reduction in percolation threshold.

The electrical conductivity is also influenced by the other internal parameter, namely, the presence of clusters. Many studies show that the presence of clusters improves the electrical conductivity only if the effective contact area between CNTs is increased, which in turn decreases the contact resistance. ${ }^{36-39}$ We observe that the amount of clusters is increased by a factor of about 3 by going from insulating $(\varphi \approx$
Electrical percolation behavior

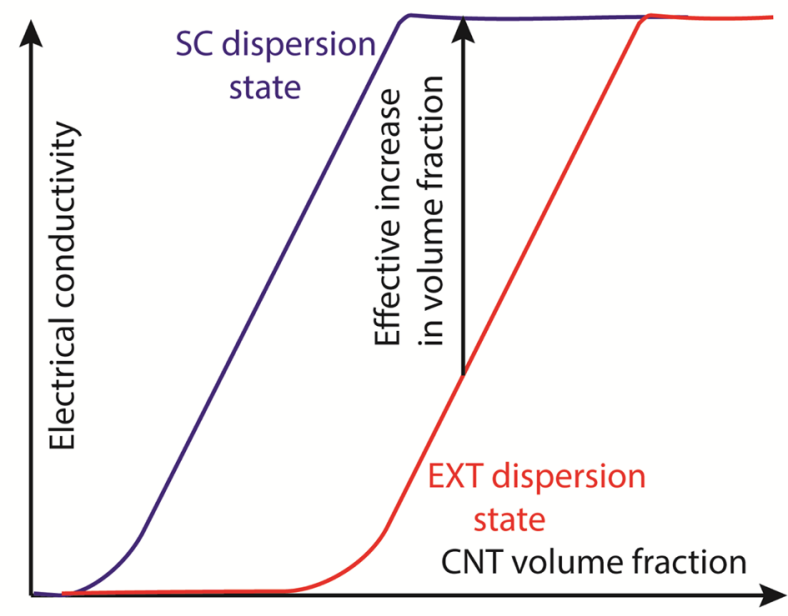

Mesoscale variations

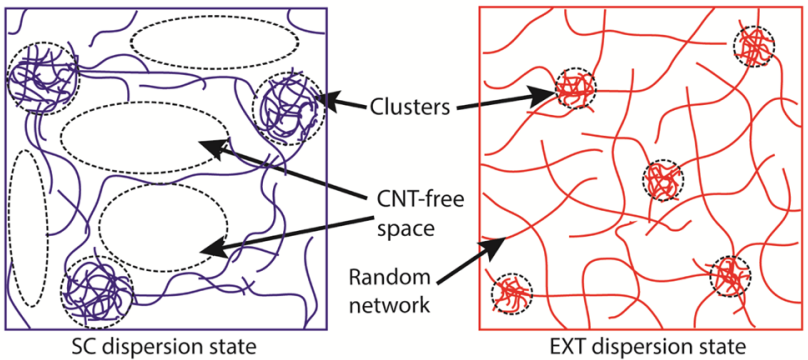

Nanoscale variations

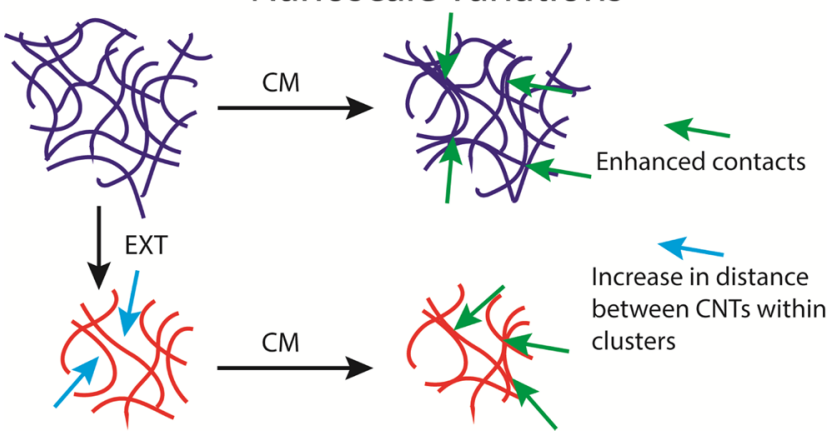

Figure 7. Schematic illustration of conductive networks of SC-based (SC and SC-CM - in blue) and EXT-based (EXT and EXT-CM - in red) samples and their percolation behavior.

$0.001)$ to percolating $(\varphi \approx 0.01)$ in both SC-CM and EXT samples (Table 2). Moreover, during compression molding, there is a certain increase in mobility, which results in stronger $\mathrm{CNT}-\mathrm{CNT}$ interactions and better CNT contacts. The drop in the measured resistance plotted as a function of CNT volume fraction is shown in Figure 9, where, to be explicit, the resistance drop due to compression molding $\left(R_{\mathrm{SC}}, R_{\mathrm{EXT}}\right)$ is calculated for both the SC-based (SC and SC-CM) and EXTbased (EXT and EXT-CM) samples, respectively, as 


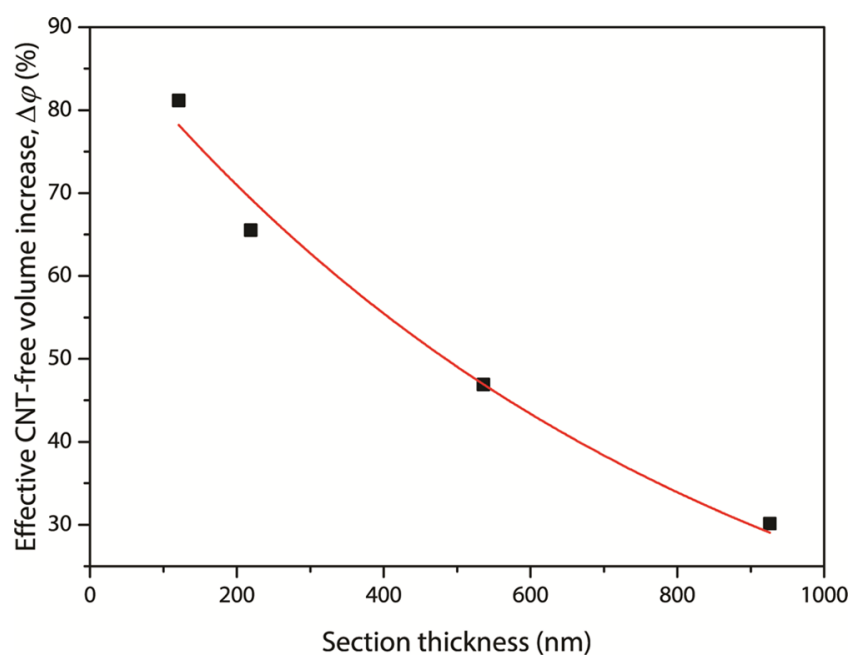

Figure 8. Effective CNT-free volume increase $\Delta \varphi(\%)$ as a function of section thickness $t(\mathrm{~nm})$. The line represents the exponential fit given by $\Delta \varphi=89.2 \exp \left(-1.18 \times 10^{-3} t\right)$ having $R^{2}=0.991$.

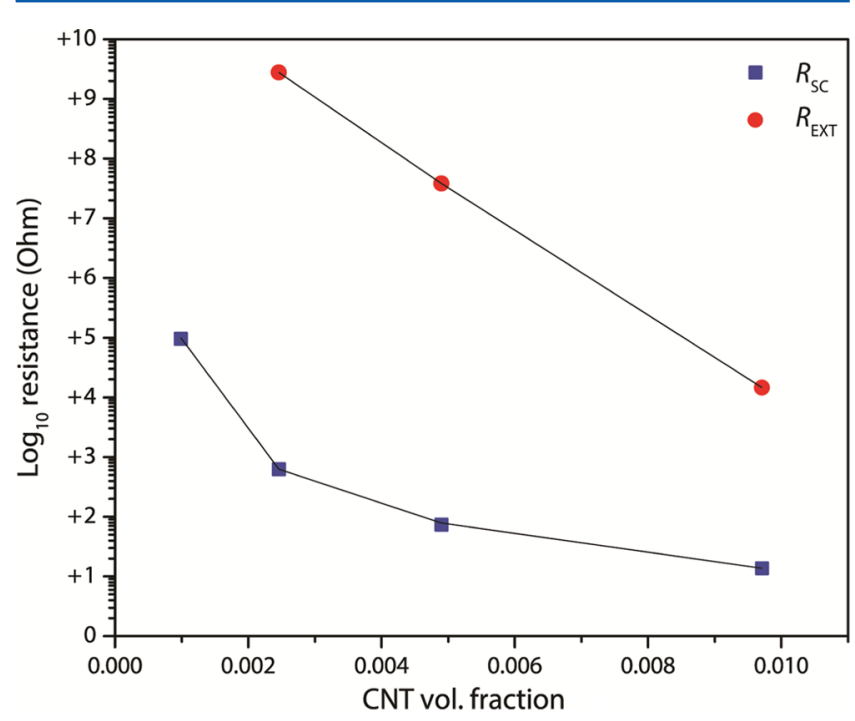

Figure 9. Resistance drop due to compression molding plotted as a function of CNT volume fraction for SC- and EXT-based samples (line drawn as a guide to the eye).

$$
\frac{1}{R_{\mathrm{SC}}}=\frac{1}{\Omega_{\mathrm{SC}-\mathrm{CM}}}-\frac{1}{\Omega_{\mathrm{SC}}} ; \frac{1}{R_{\mathrm{EXT}}}=\frac{1}{\Omega_{\mathrm{EXT}-\mathrm{CM}}}-\frac{1}{\Omega_{\mathrm{EXT}}}
$$

Almost the same pattern of resistance drop with increasing CNT concentration is observed for both types of samples, but the resistance drop is larger for EXT samples than for the SC samples, which represents the inherent difference in the mesoscale conductive network for the SC and EXT samples. For the SC samples at the percolation volume fraction $\left(\varphi_{\mathrm{c}} \approx\right.$ $0.001)$ the absolute resistance value is $\approx 9.5 \times 10^{4} \mathrm{ohm}$, while for the EXT samples at the percolation threshold $\left(\varphi_{\mathrm{c}} \approx 0.01\right)$, it is $\approx 1.5 \times 10^{4} \mathrm{ohm}$. This same order of magnitude clearly indicates that the resistance drop is induced just by the enhanced CNT contacts. Yet, the difference in the resistance drop for the EXT samples is about a factor of 6 larger than for the SC samples, but this can be related to the presence of 3 times more clusters (Table 2) as well as 10 times more CNTs. In addition, the local agglomeration process during the solvent casting itself results in CNT contacts close to the optimization point, which leaves less improvement by the compression molding process. Although the exact influence of the clusters on the resistance drop cannot be assessed, the analysis reveals the trend for both improved contacts and the presence of clusters.

\section{CONCLUSIONS}

From conductivity measurements, observed network topology, and quantitative image analysis, a detailed understanding of the CNT network formation and their electrical conductivity behavior can be obtained. The broader distribution of the local area fraction of CNTs (Figure 5) and the increase in the maximum free distance distribution of CNT-free space (Figure 6) are consistent with an inhomogeneous CNT distribution and shift the percolation threshold to a lower overall volume fraction. This leads to a local increase in volume fraction (Table 2) for the SC samples as compared to a random network and therefore to higher conductivity in SC-based samples. Local optimization of nanoscale CNT contacts is induced during compression molding (CM samples), which further decreases the percolation threshold and leads to a significantly improved electrical conductivity. In the case of EXT samples, the highly conductive inhomogeneous CNT network formed by the SC process is destroyed due to the massive shearing of the melt during extrusion and, since rearrangement of the network is limited, results in a new network topology resembling closely that of a random network. At low volume fraction of CNTs, the CNTs in the EXT samples are separated by large distances, and hence the CNT contacts experience a large resistance. Although by increasing the volume fraction of CNTs the distance between CNTs can be made smaller, no strong contacts are formed due to the shear involved during extrusion, which results in a low electrical conductivity. Upon compression molding of the EXT samples, local optimization occurs resulting in an increase in effective CNT-CNT contact area, thereby decreasing the contact resistance. Nevertheless, a resistance as low as observed for solvent casting (SC) followed by compression molding ( $\mathrm{CM}$ ) cannot be achieved as the global network topology is totally different.

Given the data and associated analysis, a possible route toward improving conductivity presents itself. We know that solvent casting provides a better conductivity at the same volume fraction but with an inhomogeneous distribution of CNTs (as evidenced by the presence of CNT-free regions). Further improvements in conductivity could be achieved by increasing the size of the CNT-free regions (thus introducing more inhomogeneity) while at the same time densifying the CNT network (thereby potentially increasing the contact number). Such an optimization might be possible by varying the evaporation rate during solvent casting or by using a different solvent. Compression molding (CM) probably will be needed, except if the polymer will creep into contacts by heating by itself in the case of a strong affinity between polymer and CNTs.

In addition to these physical insights, another important aspect from this study is to realize that microscopy and scattering analysis are complementary to each other. ${ }^{34}$ Scattering can probe larger volumes; however, since the data represent the average behavior, it is difficult to discern inhomogeneities, while microscopy probes smaller volumes and reveals local information. With the large-scale, highresolution (S)TEM extension ${ }^{34}$ as used here, the latter 
technique has made a step toward tackling these sampling limitations, and structural information based on images can be used as a basis for fitting scattering data. Moreover, the interactions during materials assembly can be studied and further exploited leading to hierarchical structures with improved functionalities. In this paper, we have critically analyzed the difference in the network topology induced during materials assembly that alters the electrical conductivity over 9 orders of magnitude. Additional analysis, like quantitative electron tomography of nanoscale contacts after each processing step, would push our understanding even further. The present study illustrates how multiscale information provides an important step toward the rational design of conductive composites.

\section{ASSOCIATED CONTENT}

\section{S Supporting Information}

The Supporting Information is available free of charge on the ACS Publications website at DOI: 10.1021/acs.jpcc.6b07458.

Experimental details; percolation analysis from various models; CNT length measurements; STEM imaging techniques; large-area STEM images; imaging representative of volume and analysis; segmentation and quantification methodology; simulation of random orientation of rods; section thickness measurements (PDF)

\section{AUTHOR INFORMATION}

\section{Corresponding Author}

*E-mail: g.dewith@tue.nl.

\section{Notes}

The authors declare no competing financial interest.

\section{ACKNOWLEDGMENTS}

The research leading to these results has received funding from the European Union Seventh Framework Programme (FP7MC-ITN) under grant agreement No. 264710. The authors would like to thank the Directorate-General for Science, Research and Development of the European Commission for financial support of the research.

\section{REFERENCES}

(1) Lekawa-Raus, A.; Patmore, J.; Kurzepa, L.; Bulmer, J.; Koziol, K. Electrical Properties of Carbon Nanotube Based Fibers and Their Future Use in Electrical Wiring. Adv. Funct. Mater. 2014, 24, 36613682.

(2) Hu, G.; Zhao, C.; Zhang, S.; Yang, M.; Wang, Z. Low Percolation Thresholds of Electrical Conductivity and Rheology in Poly(Ethylene Terephthalate) through the Networks of Multi-Walled Carbon Nanotubes. Polymer 2006, 47, 480-488.

(3) Du, F.; Scogna, R. C.; Zhou, W.; Brand, S.; Fischer, J. E.; Winey, K. I. Nanotube Networks in Polymer Nanocomposites: Rheology and Electrical Conductivity. Macromolecules 2004, 37, 9048-9055.

(4) Liu, L.; Ma, W.; Zhang, Z. Macroscopic Carbon Nanotube Assemblies: Preparation, Properties, and Potential Applications. Small 2011, 7, 1504-1520.

(5) Kyrylyuk, A. V.; Hermant, M. C.; Schilling, T.; Klumperman, B.; Koning, C. E.; van der Schoot, P. Controlling Electrical Percolation in Multicomponent Carbon Nanotube Dispersions. Nat. Nanotechnol. 2011, 6, 364-369.

(6) Sumfleth, J.; Adroher, X. C.; Schulte, K. Synergistic Effects in Network Formation and Electrical Properties of Hybrid Epoxy Nanocomposites Containing Multiwall Carbon Nanotubes and Carbon Black. J. Mater. Sci. 2009, 44, 3241-3247.
(7) Pegel, S.; Pötschke, P.; Petzold, G.; Alig, I.; Dudkin, S. M.; Lellinger, D. Dispersion, Agglomeration, and Network Formation of Multiwalled Carbon Nanotubes in Polycarbonate Melts. Polymer 2008, 49, 974-984.

(8) Hermant, M. C.; Klumperman, B.; Kyrylyuk, A. V.; van der Schoot, P.; Koning, C. E. Lowering the Percolation Threshold of Single-Walled Carbon Nanotubes Using Polystyrene/Poly(3,4-Ethylenedioxythiophene): Poly(Styrene Sulfonate) Blends. Soft Matter 2009, 5, 878-885.

(9) Kasaliwal, G. R.; Pegel, S.; Göldel, A.; Pötschke, P.; Heinrich, G. Analysis of Agglomerate Dispersion Mechanisms of Multiwalled Carbon Nanotubes During Melt Mixing in Polycarbonate. Polymer 2010, 51, 2708-2720.

(10) Kalaitzidou, K.; Fukushima, H.; Drzal, L. T. A New Compounding Method for Exfoliated Graphite-Polypropylene Nanocomposites with Enhanced Flexural Properties and Lower Percolation Threshold. Compos. Sci. Technol. 2007, 67, 2045-2051.

(11) Grossiord, N.; Wouters, M. E. L.; Miltner, H. E.; Lu, K.; Loos, J.; Mele, B. V.; Koning, C. E. Isotactic Polypropylene/Carbon Nanotube Composites Prepared by Latex Technology: Electrical Conductivity Study. Eur. Polym. J. 2010, 46, 1833-1843.

(12) Alig, I.; Pötschke, P.; Lellinger, D.; Skipa, T.; Pegel, S.; Kasaliwal, G. R.; Villmow, T. Establishment, Morphology and Properties of Carbon Nanotube Networks in Polymer Melts. Polymer 2012, 53, 4-28.

(13) Pegel, S.; Potschke, P.; Villmow, T.; Stoyan, D.; Heinrich, G. Spatial Statistics of Carbon Nanotube Polymer Composites. Polymer 2009, 50, 2123-2132.

(14) Shante, V. K. S.; Kirkpatrick, S. Introduction to Percolation Theory. Adv. Phys. 1971, 20, 325-357.

(15) Spitalsky, Z.; Tasis, D.; Papagelis, K.; Galiotis, C. Carbon Nanotube-Polymer Composites: Chemistry, Processing, Mechanical and Electrical Properties. Prog. Polym. Sci. 2010, 35, 357-401.

(16) Ghislandi, M.; Tkalya, E.; Schillinger, S.; Koning, C. E.; de With, G. High Performance Graphene- and Mwcnts-Based Ps/Ppo Composites Obtained via Organic Solvent Dispersion. Compos. Sci. Technol. 2013, 80, 16-22.

(17) Ghislandi, M.; Tkalya, E.; Marinho, B.; Koning, C. E.; de With, G. Electrical Conductivities of Carbon Powder Nanofillers and Their Latex-Based Polymer Composites. Composites, Part A 2013, 53, 145151.

(18) Bauhofer, W.; Kovacs, J. Z. A Review and Analysis of Electrical Percolation in Carbon Nanotube Polymer Composites. Compos. Sci. Technol. 2009, 69, 1486-1498.

(19) Pike, G. E.; Seager, C. H. Percolation and Conductivity: A Computer Study. I. Phys. Rev. B 1974, 10, 1421-1434.

(20) Seager, C. H.; Pike, G. E. Percolation and Conductivity: A Computer Study. II. Phys. Rev. B 1974, 10, 1435-1446.

(21) Xin, F.; Li, L.; Chan, S. H.; Zhao, J. Influences of Carbon Fillers on Electrical Conductivity and Crystallinity of Polyethylene Terephthalate. J. Compos. Mater. 2012, 46, 1091-1099.

(22) Regev, O.; ElKati, P. N. B.; Loos, J.; Koning, C. E. Preparation of Conductive Nanotube-Polymer Composites Using Latex Technology. Adv. Mater. 2004, 16, 248-251.

(23) Weber, M.; Kamal, M. R. Estimation of the Volume Resistivity of Electrically Conductive Composites. Polym. Compos. 1997, 18, $711-725$.

(24) Balberg, I. I. Tunneling and Nonuniversal Conductivity in Composite Materials. Phys. Rev. Lett. 1987, 59, 1305-1308.

(25) Kogut, P. M.; Straley, J. P. Distribution-Induced NonUniversality of the Percolation Conductivity Exponents. J. Phys. C: Solid State Phys. 1979, 12, 2151-2159.

(26) Straley, J. P. Non-Universal Threshold Behaviour of Random Resistor Networks with Anomalous Distributions of Conductances. J. Phys. C: Solid State Phys. 1982, 15, 2343-2346.

(27) Rubin, Z.; Sunshine, S. A.; Heaney, M. B.; Bloom, I.; Balberg, I. Critical Behavior of the Electrical Transport Properties in a TunnelingPercolation System. Phys. Rev. B: Condens. Matter Mater. Phys. 1999, 59, 12196-12199. 
(28) Krause, B.; Boldt, R.; Potschke, P. A Method for Determination of Length Distributions of Multiwalled Carbon Nanotubes before and after Melt Processing. Carbon 2011, 49, 1243-1247.

(29) Tung, W.-S.; Composto, R. J.; Clarke, N.; Winey, K. I. Anisotropic Polymer Conformations in Aligned Swcnt/Ps Nanocomposites. ACS Macro Lett. 2015, 4, 916-920.

(30) Du, F.; Fischer, J. E.; Winey, K. I. Effect of Nanotube Alignment on Percolation Conductivity in Carbon Nanotube/Polymer Composites. Phys. Rev. B: Condens. Matter Mater. Phys. 2005, 72, 121404.

(31) Ran, S.; Fang, D.; Zong, X.; Hsiao, B. S.; Chu, B.; Cunniff, P. M. Structural Changes During Deformation of Kevlar Fibers via On-line Synchrotron SAXS/WAXD Techniques. Polymer 2001, 42, 16011612.

(32) Schaefer, D. W.; Zhao, J.; Brown, J. M.; Anderson, D. P.; Tomlin, D. W. Morphology of Dispersed Carbon Single-Walled Nanotubes. Chem. Phys. Lett. 2003, 375, 369-375.

(33) Bauer, B. J.; Hobbie, E. K.; Becker, M. L. Small-Angle Neutron Scattering from Labeled Single-Wall Carbon Nanotubes. Macromolecules 2006, 39, 2637-2642.

(34) Gnanasekaran, K.; Snel, R.; de With, G.; Friedrich, H. Quantitative Nanoscopy: Tackling Sampling Limitations in (S)TEM Imaging of Polymers and Composites. Ultramicroscopy 2016, 160, 130-139.

(35) Marinho, B.; Ghislandi, M.; Tkalya, E.; Koning, C. E.; de With, G. Electrical Conductivity of Compacts of Graphene, Multi-Wall Carbon Nanotubes, Carbon Black, and Graphite Powder. Powder Technol. 2012, 221, 351-358.

(36) Aguilar, J. O. Influence of Carbon Nanotube Clustering on the Electrical Conductivity of Polymer Composite Films. eXPRESS Polym. Lett. 2010, 4, 292-299.

(37) Cardoso, P.; Silva, J.; Klosterman, D.; Covas, J. A.; van Hattum, F. W.; Simoes, R.; Lanceros-Mendez, S. The Influence of the Dispersion Method on the Electrical Properties of Vapor-Grown Carbon Nanofiber/Epoxy Composites. Nanoscale Res. Lett. 2011, 6, 370-374.

(38) Vigolo, B.; Coulon, C.; Maugey, M.; Zakri, C.; Poulin, P. An Experimental Approach to the Percolation of Sticky Nanotubes. Science 2005, 309, 920-923.

(39) Gnanasekaran, K.; de With, G.; Friedrich, H. On Packing, Connectivity, and Conductivity in Mesoscale Networks of Polydisperse Multiwalled Carbon Nanotubes. J. Phys. Chem. C 2014, 118, 29796-29803.

(40) Weibel, E. R. Stereological Methods in Cell Biology - Where Are We - Where Are We Going. J. Histochem. Cytochem. 1981, 29, 10431052.

(41) Underwood, E. E. Stereology, or the Quantitative Evaluation of Microstructures. J. Microsc. 1969, 89, 161-180. 